\title{
REDUCIBILITY BY MOMENTS OF LINEAR IMPULSE MARKOV DYNAMICAL SYSTEMS WITH ALMOST CONSTANT COEFFICIENTS
}

\author{
Jevgeṇijs Carkovs, Aija Pola, and Kārlis Šadurskis" \\ Department of Theory of Probability and Mathematical Statistics, Rīga Technical University, \\ Kalı̣u iela 1, Rīga, LV-1658, LATVIA \\ \# Corresponding author
}

Communicated by Jurijs Merkurjevs

This paper deals with linear impulse dynamical systems on $\mathbb{R}^{d}$ whose parameters depend on an ergodic piece-wise constant Markov process with values from some phase space $\mathbb{Y}$ and on a small parameter $\varepsilon$. Trajectories of Markov process $x(t, y) \in \mathbb{R}^{d}$ satisfy a system of linear differential equations with close to constant coefficients on its continuity intervals, while its phase coordinate changes discontinuously when Markov process switching occur. Jump sizes depend linearly on the phase coordinate and are proportional to the small parameter $\varepsilon$. We propose a method and an algorithm for choosing the base $\mathbb{B}(t, y)$ of the space $\mathbb{R}^{d}$ that provides approximation of average phase trajectories $\boldsymbol{E}\{x(t, y)\}$ by a solution of a system of linear differential equations with constant coefficients.

Key words: dynamical system, Markov process, reducibility, perturbation theory.

\section{INTRODUCTION}

In the theory of linear differential equations with variable coefficients on $\mathbb{R}^{d}$

$\frac{d x}{d t}=A(t) x$

the term "reducibility" usually means, e.g. (Fink, 1974), construction of a matrix $B(t)$ such that the equation (1) by means of substitution $\tilde{x}(t)=B(t) x(t)$ may be transformed to the equation with constant coefficients for some vector function $\tilde{x}(t)$ :

$\frac{d \tilde{x}}{d t}=\hat{A} \tilde{x}(t)$

The reducibility problem is sufficiently well studied not only for type (1) equations, but also for impulse type equations whose trajectories have jumps

$x(t)=C(t) x(t-)$

at time moments defined by some sequence $t \in S:=$ $\left\{t_{k}, k \in \mathbb{N}\right\}$, e.g., (Fink, 1974). In this paper we also analyse systems of types (1) - (3), but in our case matrices in the right-hand side of these equations are not only time but also stochastic process dependent.
We shall use the following notations and proposals:

- $\left(\Omega, \mathcal{F}, \mathcal{F}^{t}, \mathbf{P}\right)$ - filtered probability space;

- $\{y(t), t \geq 0\}-$ homogeneous right-continuous piecewiseconstant Poisson Markov process with a finite state space $\mathbb{Y}=\left\{y_{1}, y_{2}, \ldots, y_{r}\right\} \subset \mathbb{R}$

- $Q$ - infinitesimal operator of the aforementioned Markov process, given on a space of functions by the equation

$$
\begin{aligned}
Q v(y):=\lim _{s \downarrow 0} \frac{1}{s} \mathbf{E}\{v(y(t+s))- & v(t) / y(t)=y\}= \\
& =\sum_{z \in \mathbb{Y}}[v(z)-v(y)] p(y, z)
\end{aligned}
$$

with transitional probability of the embedded Markov chain $p(y, z)$ that satisfies the condition $p(y, y) \equiv 0$;

- $\sigma(Q)$ - spectrum of the infinitesimal operator (4) that can be represented as the union of sets:

$\sigma(Q)=0 \cup \sigma_{\rho}$

where $\sigma_{\rho} \subset\{\operatorname{Re} z \leq-\rho<0\}$ and 0 is an isolated spectrum point with multiplicity 1 .

This definition implies exponential ergodicity (Dynkin, 1965) of the process $\{y(t)\}$, i.e., there exists a distribution $\{\mu(y), y \in \mathbb{Y}\}$, such that all distributions of the process $y(t)$ 
converge exponentially to it for $t \rightarrow \infty$. This distribution may be obtained by solving the equation (Dynkin, 1965):

$$
\left(Q^{*} \mu\right)(z):=\sum_{y \in Y}(p(y, z)(\mu(y)-\mu(z))=0
$$

Time moments $\left\{\tau_{k}, k=0,1,2, \ldots\right\}$ of switches of the Markov process are given by the formulas (Dynkin, 1965):

$\tau_{0}=0, \mathbf{P}\left(\tau_{\mathrm{k}}-\tau_{\mathrm{k}-1}>\mathrm{t} / \mathrm{y}\left(\tau_{\mathrm{k}-1}\right)=y\right)=\exp \{-t\}, j \in \mathbb{N}, \mathrm{y} \in \mathbf{Y}, t \geq 0$

An impulse Markov dynamical system, mentioned in the title of this paper, with phase coordinates $x(t) \in \mathbb{R}^{d}$ for $t \geq 0$ has been defined on probability space $\left(\Omega, \mathcal{F}, \mathcal{F}^{t}, \mathbf{P}\right)$ by the following formulas:

- initial condition:

$$
x(0)=x
$$

- differential equation:

$$
\frac{d x}{d t}=A(t, y(t / \varepsilon), \varepsilon) x
$$

on any interval $\left(\varepsilon \tau_{j-1}<t<\varepsilon \tau_{j}\right), j \in \mathbb{N}$;

- condition of jumps:

$$
x\left(\varepsilon \tau_{j}\right)=G\left(\varepsilon \tau_{j}, y\left(\tau_{j-1}\right), \varepsilon\right) x\left(\varepsilon \tau_{j-1}\right)
$$

at time moments $t \in\left\{\varepsilon \tau_{j}, j \in \mathbb{N}\right\}$ where $\varepsilon \in\left(0, \varepsilon_{0}\right)$ is a small parameter, and matrices $A(t, y, \varepsilon), G(t, y, \varepsilon)$ may be expanded into uniformly convergent $\varepsilon$ power series:

$$
\begin{aligned}
& A(t, y, \varepsilon)=A_{0}+\varepsilon A_{1}(t, y)+\varepsilon^{2} A_{1}(t, y)+\ldots, \\
& G(t, y, \varepsilon)=I+\varepsilon^{2} C(t, y)+\ldots
\end{aligned}
$$

around zero $U:=\left\{|\varepsilon|<\varepsilon_{0}\right\}$ continuously by $t$ for all $y \in \mathbb{Y}$, $\varepsilon \in U$ and are bounded:

$$
\sup _{t, y, \varepsilon}\{\mid A(t, y, \varepsilon)\|+\| G(t, y, \varepsilon) \|\} \leq \alpha
$$

As mentioned above, to reduce type (1) equations with semi-periodic coefficients, the substitution $\tilde{x}(t)=B(t) x(t)$ may be used. In case of equations (8) - (9) the matrix of this substitution must depend on the phase coordinates of the random process with piece-wise constant trajectories with discontinuities at random moments, i.e. the substitution must be $B(t, y)$. Intervals where Markov process $y(t / \varepsilon)$ is constant are proportional to small parameter $\varepsilon$. Thus, even if one could find solution $B(t, y)$ for each interval $\varepsilon \tau_{j-1}<t<$ $\varepsilon \tau_{j}$ where process $y(t / \varepsilon)$ keeps its constant value, in order to solve the system of equations (8) - (9) on the interval of unitary length, it would be necessary to find a large number (commensurate with $\varepsilon^{-1}$ ) of solutions of differential matrix equations. Besides, the problem of how to take into consideration Markov switchings (9) still persists. Consequently, it is doubtful whether this approach can be used for practical research on the system (8) - (9).

Here we propose a method and an algorithm of construction of a system of differential equations whose solutions approximate moments of solutions of system (8) - (9). This may be done so as to take in respect exponential ergodicity (5) of Markov process and proportionality of switchings (9) to $\varepsilon^{2}$.

Let us discuss reducibility of type (1) equations with close to constant coefficients, i.e. in the case of $A(t)=A_{0}+$ $\varepsilon A_{1}(t)$. The reducibility issue for such equations was discussed in detail in the monograph (Bogoljubov et al., 1976). There we have a feasible method and an algorithm of reducibility in the case of finite basis of frequencies $\left\{v_{1}, v_{2}, \ldots, v_{\mathrm{m}}\right\}$ of semi-periodic matrix-function $A_{1}(t)$. A necessary condition of reducibility in this case is solvability of a matrix system of differential equations

$$
\begin{aligned}
& \frac{d}{d t} B(t)+A_{0}^{T} B(t)-B(t) A_{0}^{T}= \\
&=\exp \left\{i t\left(k_{1} v_{1}+k_{2} v_{2}+\ldots+k_{m} v_{m}\right)\right\}
\end{aligned}
$$

for any integer numeric vectors $\left\{k_{1}, k_{2}, \ldots, k_{m}\right\}$. It is evident that absence of resonance is necessary for existence of a bounded solution of the equation (12) (Bogoljubov et al., 1976), i.e., eigenvalues of the matrix $A_{0}$ must not be equal to any of combinations $i\left(k_{1} v_{1}+k_{2} v_{2}+\ldots+k_{m} v_{m}\right)$. In the case being discussed in the present paper the algorithm orders solution of a system of matrix equations

$$
(Q B)(t, y)+\frac{d}{d t} B(t, y)+A_{0}^{T} B(t, y)-B(t, y) A_{0}^{T}=f(t, y)
$$

However, we shall show that the equation (13) may have a solution even in the case of frequency resonance on the assumption that an infinitesimal operator of the Markov process has a spectrum of form (5), if spectrum of matrix $A_{0}$ is located in a margin $\mid \operatorname{Re} \lambda \leq \alpha<\rho / 2$ and the right-hand side of this equation, averaged with respect to the invariant measure $\mu$, is equal to zero

$\hat{f}(t)=\sum_{y \in \mathbb{Y}} f(t, y) \mu(y)=0$

Here and further we shall use a "cap" symbol above a letter " $ڤ$ " to denote averaging with respect to measure $\mu(y)$.

\section{Notations and assumptions}

Besides averaging with respect to the invariant measure we will have to deal with the averaging operator with respect to time

$\bar{u}:=\lim _{T \rightarrow \infty} \frac{1}{T} \int_{0}^{T} u(t) d t$

This operator is defined on the space of such functions $\mathbb{U} \subset \mathbb{C}(\mathbb{R})$ for which the limit (14) exists and the following condition is true:

$\exists K>0: \sup _{r>0} \int_{0}^{t}|U(s)-\bar{u}| d s<K\|u\|$

Let us remind that the Markov process $\{y(t)\}$ is exponentially ergodic (Dynkin, 1965). This means that distributions 
of random variables $y(t)$ exponentially tend to distribution $\mu(y)$ if $t \rightarrow \infty$ for any distribution of initial value $y(0)$. This provides that:

$$
\begin{aligned}
\exists M>0, \forall v \in \mathbb{B}(\mathbb{Y}), \forall t \geq 0: \| \mathbf{E}\{v(y(t)) / y(0) & =y\}-\hat{v} \| \leq \\
& \leq M\|v\| e^{-\rho t}
\end{aligned}
$$

and therefore

$$
\hat{v}:=\lim _{T \rightarrow \infty} \frac{1}{T} \int_{0}^{T} v(y(t)) d t
$$

Let us denote by $\mathbb{D}$ a set of right-continuous by $t$ functions $u(t, y)$ whose average with respect to measure $\{\hat{u}(t), t \geq 0\}$ is an element of the space $\mathbb{U}$. Let us define the following operator on this space:

$(\mathbf{R} u)(t, y):=\int_{t}^{\infty}\left[\mathbf{E}_{t, y}\{u(s, y(s))\}-\hat{u}(s)\right] d s+\int_{0}^{t}[\hat{u}(s)-\overline{\hat{u}}] d s$

where

$\overline{\hat{u}}=\lim _{T \rightarrow \infty} \frac{1}{T} \int_{0}^{T} \sum_{y \in \mathbb{Y}} u(t, y) \mu(y) d t$

Subindices at the sign of an expectation operator mean that the expectation has been taken under condition $y(t)=y$, i.e., $\mathbf{E}_{t, y}\{\bullet\}:=\mathbf{E}\{\bullet / y(t)=y\}$. We see that operator $\mathbf{R}$ determines a solution of the equation

$\left\{\frac{d}{d x}+Q\right\}(\mathbf{R} u)(t, x)=-u(t, y)+\overline{\hat{u}}$

This means that it is a potential of the operator $\frac{d}{d t}+Q$ on the subspace of functions for which $\overline{\hat{u}}=0$ is fulfilled. Let us denote by $\mathbb{G}$ a space of vector functions continuously differentiable at $\mathrm{t} \in \mathbb{R}$ and dependent on $\mathrm{y} \in \mathbb{Y}$. Using the Markov property, let us find the right derivative in the system (8) (9) for the expectation $\mathbf{E}\left\{(g(t, y(t)))^{T} x(t)\right\}$ with $g \in \mathbb{G}$ :

$$
\begin{gathered}
\left.\frac{d}{d t} E\left\{(g(t, y(t)))^{T} x(t)\right\}\right|_{y(t)=Y, x(t)=x}= \\
=\lim _{s \downarrow 0} \frac{1}{s}\left[\mathbf{E}\left\{(g(t+s, y(t+s)))^{T} x(t+s) / y(t)=y, x(t)=x\right\}-\right. \\
\left.-E\left\{(g(t, y))^{T}\right]=[\mathcal{L}(\varepsilon) g)(t, y)\right]^{T} x
\end{gathered}
$$

where operator $\mathcal{L}(\varepsilon)$ may be represented as a uniformly convergent series of operators:

$$
\begin{aligned}
\mathcal{L}(\varepsilon)=\mathcal{L}_{0}+\varepsilon \mathcal{L}_{1}+\varepsilon^{2} \mathcal{L}_{2}+\ldots \\
\left.\left(\mathcal{L}_{0} g\right)(t, y):=\left(\left\{\frac{d}{d t}+Q\right\} g\right)(t, y)\right)+\left(A_{0}\right)^{T} g(t, y) \\
\left(\mathcal{L}_{1} g\right)(t, y):=\left(A_{1}(t, y)\right)^{T} g(t, y)+ \\
+(C(t, y))^{T} \int_{\mathbb{Y}}[g(t, z)-g(t, y)] p(y, d z) \\
\left(\mathcal{L}_{2} g\right)(t, y):=\left(A_{2}(t, y)\right)^{T} g(t, y)
\end{aligned}
$$

and so on. Here and further symbol $T$ as an upper index denotes transposition both for a vector and a matrix. Let us rewrite formula (20) into the form of an operator equation

$\frac{d}{d t} \mathbf{E}\{g(t, y(t))\}=\mathbf{E}[\{(\varepsilon) g](t, y(t))\}$

and let us examine properties of the operator family (21) on the space $\mathbb{G}$ using the method and algorithm of perturbation theory proposed in monograph (Kato, 1980). By definition (22) operator $\mathcal{L}_{0}$ may be presented in the form of tensor sum

$L_{0}=\left\{\frac{d}{d t}\right\} \oplus Q \oplus A_{0}^{T}$

Differential operator $\frac{d}{d t}$ acts on the space of almost periodical functions with the finite set of frequencies $\left\{v_{1}, v_{2}, \ldots, v_{m}\right\}$, operator $Q$ acts on the space $\mathbb{B}(\mathbb{Y})$, and matrix $A_{0}^{T}$ defines an operator on the space $\mathbb{R}^{d}$. Therefore (Kato, 1980), any point of spectrum $\lambda \in \sigma\left(\mathcal{L}_{0}\right)$ may be represented as a sum of spectral points of the operators $\frac{d}{d t}$ and $Q$ and matrix $A_{0}$ :

$$
\begin{aligned}
\sigma\left(\mathcal{L}_{0}\right)=\left\{\lambda=\lambda_{1}+\lambda_{2}+\lambda_{3}: \lambda_{1}\right. & \in \sigma\left(\frac{d}{d t}\right), \\
\lambda_{2} & \left.\in \sigma(Q), \lambda_{3} \in \sigma\left(A_{0}\right)\right\}
\end{aligned}
$$

Let us denote by $\mathbf{P}_{\rho}$ a projector corresponding to the part of the spectrum $\sigma_{\rho}$ of the operator $Q$, and by $\mathbf{P}_{0}$ a supplementary spectral projector corresponding to isolated spectral point 0 with multiplicity of 1 . By definition the differential operator on the space of almost periodic functions with a finite set of frequencies $\left\{v_{1}, v_{2}, \ldots, v_{m}\right\}$ has pure imaginary spectrum with a point wise base $\left\{k_{1} v_{1}+k_{2} v_{2} \ldots+k_{m} v_{m}\right\}$, where $\left\{k_{1}, k_{2}, \ldots, k_{m}\right\}$ is an arbitrary set of numbers $k_{j} \in \mathbb{Z}$, $j=1,2, \ldots, m$ (Kato, 1980). Spectrum of the shrinkage $Q \mathbf{P}_{\sigma}$ of the operator $Q$ onto subspace $\mathbf{P}_{\sigma} \mathbf{G}$ by the definition (Kato, 1980) has been located on half-plane $\operatorname{Re} \lambda<-\rho$, and spectrum of the matrix $A_{0}$ lies in $|\boldsymbol{R e} \lambda| \leq \alpha<\rho / 2$. Thus, on the subspace $\mathbf{P}_{\sigma} \mathbb{G}$ the spectrum of the operator $\frac{d}{d t}+Q \mathbf{P}_{\sigma}$ is located in the left-hand half-plane $\operatorname{Re} \lambda<0$. The spectrum $\sigma\left(\mathcal{L}_{0}\right)$ may be expanded into two non-intersecting sets:

$\sigma\left(\mathcal{L}_{0}\right)=\sigma\left(\frac{d}{d t}+Q \mathbf{P}_{\rho}\right) \cup \sigma\left(A_{0}\right)$

Using results of perturbation theory for linear operators (Kato, 1980), it is possible to expand spectrum $\sigma(\mathcal{L}(\varepsilon)$ ) into two non-intersecting subsets

$\sigma(\mathcal{L}(\varepsilon))=\sigma_{0}(\varepsilon) \cup \sigma_{\rho}(\varepsilon)$

for sufficiently small values of the parameter $\varepsilon$. Additionally, $\lim _{\varepsilon \rightarrow 0} \sigma_{\rho}(\varepsilon)=\sigma\left(\frac{d}{d t}+Q \mathbf{P}_{\rho}\right)$ and $\lim _{\varepsilon \rightarrow 0} \sigma_{0}(\varepsilon)=\sigma\left(A_{0}\right)$.

As the set $\sigma\left(A_{0}\right)$ contains a finite number of points, there exists a positive number $\varepsilon_{0}$ such that for all $\varepsilon \in\left(0, \varepsilon_{0}\right)$

$\sigma_{\rho}(\varepsilon) \subset\{\boldsymbol{R e} \lambda \leq-\gamma<-\alpha / 2<0\}$

Let us denote $\mathbf{P}_{0}(\varepsilon)$ and $\mathbf{P}_{\rho}(\varepsilon)$ spectral projectors corresponding to the parts of spectrum $\sigma_{0}(\varepsilon)$ and $\sigma_{\rho}(\varepsilon)$ correspondingly on the subspace $\mathbb{G}$. By the definition (Kato, 
$1980)$, projector $\mathbf{P}_{0}(\varepsilon)$ is an analytical function of the parameter $\varepsilon$ in some neighbourhood of the point $\varepsilon=0$, and $\lim \mathbf{P}(\varepsilon)=I$, where $I$ is an unitary $d \times d$ matrix. Hence, there

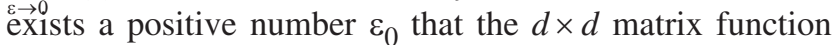

$\mathbb{B}(t, y, \varepsilon):=\left(\mathbf{P}_{0}(\varepsilon) I\right)(t, y)$

is non singular for all $\varepsilon \in\left(0, \varepsilon_{0}\right), t \in \mathbb{R}$ and $y \in \mathbb{Y}$. This matrix may be chosen as a basis on the subspace $\mathbb{R}^{d}$.

\section{Asymptotic reducibility in the mean}

Let us denote by $\mathbb{H}$ the space of $d \times d$ matrix functions that are almost periodic in $t$, having the finite base $\left\{v_{1}, v_{2}, \ldots, v_{m}\right\}$ and measurable with respect of $y \in \mathbb{Y}$. Let us continue operators (22), (23), and (24) onto this space, defining their effect on matrix functions column-wise. Similarly, following the monograph (Bogoljubov et al., 1976), let us solve the problem of reducibility of the system (8) - (9) in the mean using a base (30) on the space $\mathbb{R}^{d}$ that is analytical by parameter $\varepsilon$ and which was discussed in the previous section. Let us prove that there exist:

- independent of $t$ and $y$, analytical by $\varepsilon$ matrix function

$\Lambda(\varepsilon):=\Lambda_{\mathrm{o}}+\varepsilon \Lambda_{1}+\varepsilon^{2} \Lambda_{2}+\ldots$

- analytical by $\varepsilon$ matrix function

$\mathbb{B}(t, y, \varepsilon):=I+\varepsilon \mathbf{B}_{1}(t, y)+\varepsilon^{2} \mathbf{B}_{2}(t, y)+\ldots$

that

$\left\{\left[\left(\mathcal{L}_{0}+\varepsilon \mathcal{L}_{1}+\varepsilon^{2} \mathcal{L}_{2}+\ldots\right)\right]\right\}\left\{I+\varepsilon \mathbf{B}_{1}(t, y)+\varepsilon^{2} \mathbf{B}_{2}(t, y)+\ldots\right\}=$

$=\left\{I+\varepsilon \mathbf{B}_{1}(t, y)+\varepsilon^{2} \mathbf{B}_{2}(t, y)+\ldots\right\}\left\{\Lambda_{\mathrm{o}}+\varepsilon \Lambda_{1}+\varepsilon^{2} \Lambda_{2}+\ldots\right\}^{T}$

If we put $\varepsilon=0$ into the equation (33) we have:

$\mathcal{L}_{0} I=A_{0}^{T} I+\Lambda_{0}^{T}$

which implies $\Lambda_{0}=A_{0}$. Furthermore, equalising expressions with the same powers of the parameter $\varepsilon$, we obtain a system of equations:

$$
\begin{aligned}
\left.\left(\left\{\frac{d}{d t}+Q\right\} \mathbf{B}_{1}\right)(t, y)\right) & +A_{0}^{T} \mathbf{B}_{1}(t, y)-\mathbf{B}_{1}(t, y) A_{0}^{T}= \\
= & -A_{1}^{T}(t, y) I+\Lambda_{1}^{T}
\end{aligned}
$$

$\left.\left(\left\{\frac{d}{d t}+Q\right\} \mathbf{B}_{2}\right)(t, y)\right)+A_{0}^{T} \mathbf{B}_{2}(t, y)-\mathbf{B}_{2}(t, y) A_{0}^{T}=$

$$
\begin{aligned}
= & -A_{1}^{T}(t, y) \mathbf{B}_{1}(t, y)-A_{2}^{T}(t, y) I- \\
& -C_{1}^{T}(t, y)\left(Q \mathbf{B}_{1}\right)(t, y)+\mathbf{B}_{1} \Lambda_{1}^{T}+\Lambda_{21}^{T}
\end{aligned}
$$

and so on. Let us define an operator

$\mathcal{A} g:=A_{0}^{T} g-g A_{0}^{T}$

for the matrix $g \in \mathbb{H}$ and let us study the spectral properties of the operator
$\mathcal{G}=\left\{\frac{d}{d t}\right\}+Q+\mathcal{A}$

which we have on the right-hand side of equations (35) (36). We may write operator (38) in the form of tensor sum

$\mathcal{G}=\left\{\frac{d}{d t}\right\} \oplus Q \oplus \mathcal{A}$

and therefore (Kato, 1980)

$\sigma(\mathcal{G})=\left\{\lambda_{1}+\lambda_{2}+\lambda_{3}: \lambda_{1} \in \sigma\left(\frac{d}{d t}\right), \lambda_{2} \in \sigma(Q), \lambda_{3} \in \sigma(\mathcal{A})\right\}$ (39)

Moreover, from the definition (37) follows the formula

$\sigma(\mathcal{A})=\left\{\lambda_{1}-\lambda_{2}: \lambda_{1} \in \sigma\left(A_{0}\right), \lambda_{2} \in \sigma\left(A_{0}\right)\right.$

As described, the spectrum of the matrix $A_{0}$ is located into the margin $|\boldsymbol{R e} \lambda| \leq \alpha<\rho / 2$ and the differential operator on the space of almost periodic functions with a finite set of frequencies $\left\{v_{1}, v_{2}, \ldots, v_{m}\right\}$ by definition has a pure imaginary spectrum with a point-wise base $\left(k_{1} v_{1}, k_{2} v_{2}, \ldots, k_{3} v_{m}\right)$ where $\left\{k_{1}, k_{2}, \ldots, k_{m}\right\}$ is an arbitrary set of numbers $k_{j} \in \mathbb{Z}, j=$ 1,2,..,m (Bogoljubov et al., 1976).

Spectrum of the shrinkage $Q \mathbf{P}_{\rho}$ of the operator $Q$ onto subspace $\mathbf{P}_{\rho} \mathbb{H}$ by definition is located on half-plane $|\boldsymbol{R e} \lambda|<-\rho$, and spectrum of the operator $\mathcal{A}$ lies in $|\boldsymbol{R e} \lambda| \leq 2 \alpha<\rho$. Thus, on the subspace $\mathbf{P}_{\rho} \mathbb{H}$ the spectrum of the operator from the left-hand side of the equation (35) is located to the left of the imaginary axis. Based on these remarks we can look for a solution $\mathbf{B}_{1}$ of the equation (35) in the form of the sum of two projections

$\mathbf{B}_{1}=\tilde{\mathbf{B}}_{1}+\hat{\mathbf{B}}_{1}$

where $\widetilde{\mathbf{B}}_{1}=\mathbf{P}_{\rho} \mathbf{B}_{1}$ and $\hat{\mathbf{B}}_{1}=\mathbf{P}_{0} \mathbf{B}_{1}$. Since projector $\mathbf{P}_{0}$ corresponds to 0 point of spectrum of the operator $Q$, we have $Q \hat{\mathbf{B}}_{1}=Q \mathbf{P}_{0} \mathbf{B}_{1}=0$. The matrix functions $\widetilde{\mathbf{B}}_{1}$ and $\hat{\mathbf{B}}_{1}$, defined above, can be found from two independent equations:

$$
\begin{aligned}
& \frac{d}{d t} \tilde{\mathbf{B}}_{1}(t, y)+Q \tilde{\mathbf{B}}_{1}(t, y)+A_{0}^{T} \widetilde{\mathbf{B}}_{1}(t, y)-\tilde{\mathbf{B}}_{1}(t, y) A_{0}^{T}= \\
& =-\left(A_{1}^{T}(t, y)-\hat{A}_{1}^{T}(t)\right) \\
& \frac{d}{d t} \hat{\mathbf{B}}_{1}(t)+A_{0}^{T} \hat{\mathbf{B}}_{1}(t)+\hat{\mathbf{B}}_{1}(t) A_{0}^{T}=-\hat{A}_{1}^{T}(t)-\Lambda_{1}^{T}
\end{aligned}
$$

The right-hand side of the equation (40), based on its construction, is an element of the space $\mathbf{P}_{\rho} \mathbb{H}$, and the spectrum of the operator from the left-hand side of the equation does not contain the 0 point. Therefore, we can find a solution to this equation and proceed to the equation (41). Difficulties of solving this equation are described in detail in (Bogoljubov et al., 1976). A necessary condition for the existence of a solution of this equation is the equality

$$
\Lambda_{1}=\overline{\hat{A}_{1}}=\lim _{T \rightarrow \infty} \frac{1}{T} \int_{0}^{T} \sum_{y \in \mathbb{Y}} A_{1}(t, y) \mu(y) d t
$$


The sufficient condition of existence of a solution with $\Lambda_{1}:=\hat{A}_{1}$ is:

$\lim _{T \rightarrow \infty} \frac{1}{T} \int_{0}^{T}\left(\hat{A}_{1}^{T}(t)-\Lambda_{1}^{T}\right) \exp \{i \omega t\} d t=0$

for any

$\omega \in\left\{\operatorname{Im}\left(\lambda_{1}-\lambda_{2}\right): \lambda_{1} \in \sigma\left(A_{0}\right), \lambda_{2} \in \sigma\left(A_{0}\right)\right\}$

Insertion of $\Lambda_{1}:=\overline{\hat{A_{1}}}$ into the equation (41) allows applying a method of construction of the solution proposed in monograph (Bogoljubov et al., 1976). This method allows to find a bounded almost periodic matrix function $\hat{\mathbf{B}}_{1}(t)$ from the equation (41), insert it into the equation (40) and find an almost periodic function $\widetilde{\mathbf{B}}_{1}(t, y)$. At the next step, it is necessary to make a substitution $\mathbf{B}_{1}(t, y)=\widetilde{\mathbf{B}}_{1}(t, y)+\hat{\mathbf{B}}_{1}(t)$ in the equation (36) and to repeat the previous procedure of finding a solution using a method of projecting onto subspaces $\mathbf{P}_{0} \mathbb{H}$ and $\mathbf{P}_{\rho} \mathbb{H}$.

Remark 1. If matrices (10) - (11) are not time dependent, the basis matrix function (32) is not time dependent either, i.e., $\mathbb{B}(t, y, \varepsilon)=\mathbf{B}(y, \varepsilon)$. Therefore, we may put $\hat{B}_{j}(y) \equiv 0$ for any $j \in \mathbb{N}$. Let us illustrate this for the example of the system of equations (10) - (11), which in this case has the form:

$\left.Q \tilde{\mathbf{B}}_{1}(y)+A \tilde{\mathbf{B}}_{1}(y)=-A_{1}^{T}(y)-\Lambda_{1}\right)$,

$A_{0}^{T} \hat{\mathbf{B}}_{1}-\hat{\mathbf{B}}_{1} A_{0}^{T}=0$

where $\mathcal{A} \tilde{\mathbf{B}}_{1}(y)=A_{0}^{T} \tilde{\mathbf{B}}_{1}(y)-\tilde{\mathbf{B}}_{1}(y) A_{0}^{T}$. In view of the assumptions made with respect to the spectrum of the matrix $A_{0}$, all points of the spectrum of the finite-dimensional operator $\mathcal{A}+Q$ have negative real parts, and operators $\mathcal{A}$ and $Q$ commute. Putting $\Lambda_{1}=\hat{A}_{1}^{T}(y)$ we find a (unique) solution $\widetilde{\mathbf{B}}_{1}(t, y) \in \mathbf{P}_{\rho} \mathbb{H}$ of the equation (44):

$\widetilde{\mathbf{B}}_{1}(t)=\int_{0}^{\infty} e^{t A_{0}^{T}}\left\{e^{t Q}\left(A_{1}^{T}(y)-\Lambda_{1}\right)\right\} e^{-t A_{0}^{T}} d t$

and as the solutions of equation (45) we choose the zero matrix.

Remark 2. If $A_{0}=0$, we have the operator $\frac{d}{d t}+Q$ in the left-hand side of the equations of type (35) - (36). Therefore, all the matrices $\left\{\Lambda_{j}, j \in \mathbb{N}\right\}$ can be found by averaging the right-hand sides of equations (35) and (36), etc. with respect to time and invariant measure. Then matrices $\mathbf{B}_{j}(t, y)$ can be found using the resolvent operator (18). For example, for the equation (35) if $A_{0}=0$ we have:

$\left(\left\{\frac{d}{d t}+Q\right\} \mathbf{B}_{1}\right)(t, y)=-A_{1}^{T}(t, y) I+\Lambda_{1}^{T}$

It is necessary to insert
$\Lambda_{1}=\overline{\hat{A}_{1}}=\lim _{T \rightarrow \infty} \frac{1}{T} \int_{0}^{T} \sum_{y \in \mathbb{Y}} A_{1}(t, y) \mu(y) d t$

and then

$\mathbf{B}_{1}(t, y)=\mathbf{R}\left\{\left(A_{1}^{T}(t, y)-\overline{\hat{A}_{1}}\right)\right\}$

Our aim is to analyse the expectation of a solution $\{x(t, s, y)$, $t \geq s\}$ of the system (8) - (9) with the initial condition $x(s)=x, y(s)=y$. By definition, this solution is a linear function of $x$, i.e., $x(t, s, y)=X(t, s, y) x$, where $X(t, s, y)$ is the matrix solution of (8) - (9) with the initial condition $X(s, s, y) \equiv I$. Therefore, the equation (20) can be rewritten in the form

$$
\begin{aligned}
\frac{d}{d t} \mathbf{E}\left\{[X(t, s, y)]^{T}\right. & \mathbb{B}(t, y(t), \varepsilon)\}=\mathbf{E}\{(\mathcal{L}(\varepsilon) B)(t, y(t), \varepsilon)\}= \\
= & \mathbf{E}\left\{[X(t, s, y)]^{T} \mathbb{B}(t, y(t), \varepsilon)\right\} \Lambda^{T}(\varepsilon)
\end{aligned}
$$

for a matrix function (32).

Therefore

$\mathbf{E}\left\{\mathbb{B}^{T}(t, y(t), \varepsilon) X(t, s, y(s)) x(s)\right\}=$

$$
=e^{(t-s) \Lambda(\varepsilon)} \mathbf{E}\left\{\mathbb{B}^{T}(t, y(t), \varepsilon) x(s)\right\}
$$

for all $t \geq s, x(s) \in \mathbb{R}^{d}$ and $y(s) \in \mathbb{Y}$. If the initial values $x(s)$ and $y(s)$ are independent random variables, then

$\mathbf{E}\left\{\mathbb{B}^{T}(s, y(s), \varepsilon) x(s)\right\}=\mathbf{E}\left\{\mathbb{B}^{T}(s, y(s), \varepsilon)\right\} \mathbf{E}\{x(s)\}$

Under the assumption of a stationarity of the Markov process $\{y(t), t \in \mathbb{Z}\}$ a random variable $y(s)$ has a distribution $\mu(y)$. As the basis defined above is a continuous almost periodic function of $t \in \mathbb{R}$, this basis can be represented as the sum of

$\mathbb{B}(t, y(t), \varepsilon)=I+\varepsilon \breve{\mathbf{B}}(t, y(t), \varepsilon)$

where the matrix

$\breve{\mathbf{B}}(t, y(t), \varepsilon)=\mathbf{B}_{1}(t, y(t), \varepsilon)+\varepsilon \mathbf{B}_{2}(t, y(t))+\ldots$

is bounded by some constant $M$ :

$\|\breve{\mathbf{B}}(t, y, \varepsilon)\| \leq M$

for any $t \in \mathbb{R}, y \in \mathbb{Y}, \varepsilon \in\left(0, \varepsilon_{0}\right)$ and for sufficiently small $\varepsilon_{0}>0$. Therefore, considering the above remark concerning the solution of averaged with respect to measure $\mu(y)$ equations of the form (41), we can rewrite this formula as a more simple equality

$\mathbf{E}\left\{\mathbb{B}^{T}(t, y(t), \varepsilon) x(t)\right\}=e^{(t-s) \Lambda(\varepsilon)} m(s)$

for any $t \geq s$, where

$$
\begin{aligned}
m(s)=\mathbf{E}\left\{\mathbb{B}^{T}(s, y(s), \varepsilon) x(t)\right\} \mathbf{E}\{x(s)\}= & \\
= & \mathbf{E}\{x(s)\}+\varepsilon \sum_{y \in \mathbb{Y}} \breve{\mathbf{B}}(t, y, \varepsilon) \mu(y)
\end{aligned}
$$

is some point from $\varepsilon$ - neighbourhood of the first moment $\mathbf{E}\{x(s)\}$. From (48) it is easy to obtain the inequality 


$$
\begin{aligned}
(1+\varepsilon M)^{-1}\left\|e^{(t-s) \Lambda(\varepsilon)} m(s)\right\| & \leq \| \mathbf{E}\{x(s) \| \leq \\
& \leq(1+\varepsilon M)^{-1}\left\|e^{(t-s) \Lambda(\varepsilon)} m(s)\right\|
\end{aligned}
$$

for any $m(s)$ from $\varepsilon-$ neighbourhood of the first moment $\mathbf{E}\{x(s)\}$. This means that the first moment $\mathbf{E}\{x(t)\}$ of the solution of (8) - (9) with the initial moment $\mathbf{E}\{x(s)\}$ can be approximated by the solution of equation

$\frac{d z}{d t}=\Lambda(\varepsilon) z$

with the initial condition $z(s)=\mathbf{E}\{x(s)\}$. The Lyapunov exponents (Skokos, 2010) of the first moments of the solutions of system (8) - (9) match with the Lyapunov exponents of equations with constant coefficients (50), i.e.,

$\lim _{t \rightarrow \infty} \frac{1}{t} \ln \mid \mathbf{E}\{x(t)\} \in\{\mathbf{R e} \lambda, \lambda \in \sigma(\Lambda(\varepsilon))\}$

\section{Example}

Let us examine the equation of the second order

$\ddot{x}+(1+\varepsilon y(t / \varepsilon) \sin (2 t)) x=0$

as an example. Here $y(t)$ is the so called "switching" Poisson process with two states $\mathbb{Y}=\{-1,1\}$ with an embedded Markov chain, given by its transitional probabilities

$\mathbf{P}(y(t)=-1 / y(t-)=1)=1), \mathbf{P}(y(t)=1 / y(t-)=-1)=1)$.

This process has a generating operator

$Q v(1)=0.5[v(1)-v(-1)] ; Q v(-1)=0.5[v(-1)-v(1)]$

and an invariant measure $\mu(y)=\{0.5,0.5\}$. Transforming the equation (52) to a matrix form, we obtain

$\frac{d}{d t} \vec{x}=\left\{A_{0}-\varepsilon A_{1}(t, y(t / \varepsilon))\right\} \vec{x}$

where

$A_{0}=\left(\begin{array}{cc}0 & 1 \\ -1 & 0\end{array}\right) ; A_{1}(t, y)=\left(\begin{array}{ll}0 & 1 \\ 1 & 0\end{array}\right) y \sin (2 t)$

It is convenient to use a substitution

$\vec{x}(t)=e^{t A_{0}} \vec{u}(t)=\left(\begin{array}{cc}\cos t & \sin t \\ -\sin t & \cos t\end{array}\right) \vec{u}(t)$

and transform (54) into form

$\frac{d}{d t} \vec{u}=-\varepsilon A_{1}^{(1)}(t, y) \vec{u}$

with

$A_{1}^{(1)}(t, y):=-\frac{y}{4}\left(\begin{array}{cc}1-\cos (4 t) & 2 \sin (2 t)+\sin (4 t) \\ -2 \sin (2 t)+\sin (4 t) & -1+\cos (4 t)\end{array}\right)$

System (35) in the case of (56) has a form:

$$
\begin{aligned}
\frac{d}{d t} \mathbf{B}_{1}(t,-1)+0.5\left(\mathbf{B}_{1}(t,-1)-\mathbf{B}_{1}(t, 1)\right) & \\
& =-\left(A_{1}^{(1)}(t,-1)\right)^{T} I+\Lambda_{1}^{T}
\end{aligned}
$$

$\frac{d}{d t} \mathbf{B}_{1}(t, 1)+0.5\left(\mathbf{B}_{1}(t, 1)-\mathbf{B}_{1}(t,-1)\right)=$

$$
=-\left(A_{1}^{(1)}(t, 1)\right)^{T} I+\Lambda_{1}^{T}
$$

where

$\mathbf{B}_{1}(t, y):=\left(\begin{array}{ll}b_{11}^{(1)}(t, y) & b_{21}^{(1)}(t, y) \\ b_{12}^{(1)}(t, y) & b_{2}^{(1)}(t, y)\end{array}\right)$

Let us rewrite this system of equations into a useful form $\frac{d}{d t} \mathbf{B}_{1}(t,-1)+\left(\mathbf{B}_{1}(t, 1)\right)=$

$$
=-\left(A_{1}^{(1)}(t,-1)\right)^{T}-\left(A_{1}^{(1)}(t, 1)\right)^{T}+2 \Lambda_{1}^{T}
$$

$\left\{\frac{d}{d t}+I\right\} \mathbf{B}_{1}(t, 1)-\left(\mathbf{B}_{1}(t,-1)\right)=$

$$
=-\left(A_{1}^{(1)}(t, 1)\right)^{T}+\left(A_{1}^{(1)}(t,-1)\right)^{T}
$$

Considering that

$\hat{A}_{1}^{(1)}(t):=\frac{1}{2} A_{1}^{(1)}(t,-1)+\frac{1}{2} A_{1}^{(1)}(t, 1) \equiv 0$

it follows that $\Lambda_{1}=0$ and from the equation (59) we obtain:

$\mathbf{B}_{1}(t,-1)+\mathbf{B}_{1}(t, 1)=\mathrm{const}$

Let us remind that by the definition of the basis the following equality has to be fulfilled:

$\mathbf{P}_{0} \mathbf{B}_{0}:=\frac{1}{2}\left(\mathbf{B}_{1}(t,-1)+\mathbf{B}_{1}(t, 1)\right)=0$

and therefore a zero constant should be put into the formula (61). It follows that $\mathbf{B}_{1}(t, y)=y \hat{\mathbf{B}}_{1}(t)$. Then from formula (60) we can obtain the equation

$\left\{\frac{d}{d t}+I\right\} \hat{\mathbf{B}}_{1}(t)=\frac{1}{4} \Gamma(t)$

where

$\Gamma(t)=\left(\begin{array}{cc}1-\cos (4 t) & -2 \sin (2 t)+\sin (4 t) \\ 2 \sin (2 t)+\sin (4 t) & -1+\cos (4 t)\end{array}\right)$

It is obvious that the equation (62) has a unique bounded periodic solution, which can be found in the form of improper integral:

$\hat{\mathbf{B}}_{1}(t)=\frac{1}{4} e^{-t} \int_{-\infty}^{t} e^{s} \Gamma(s) d s=\frac{1}{4} \int_{0}^{\infty} e^{s} \Gamma(t-s) d s$

After integration we have: 
$\hat{\mathbf{B}}_{1}(t):=\left(\begin{array}{ll}g_{11}(t) & g_{21}(t) \\ g_{12}(t) & g_{22}(t)\end{array}\right)$

where

$g_{11}(t)=-\frac{1}{68}(-17+\cos (4 t)+4 \sin (4 t))$,

$g_{12}(t)=-\frac{1}{340}(68 \cos (2 t)+20 \cos (4 t)-34 \sin (2 t)-5 \sin (4 t))$

$g_{21}(t)=-\frac{1}{340}(-68 \cos (2 t)+20 \cos (4 t)+34 \sin (2 t)-$ $-5 \sin (4 t))$

$g_{22}(t)=-\frac{1}{68}(17-\cos (4 t)-4 \sin (4 t))$

The system of equations (36) for our example is as follows: $\frac{d}{d t}\left(\mathbf{B}_{2}(t,-1)+\mathbf{B}_{2}(t, 1)\right)=2 \Lambda_{2}^{T}-$

$$
\left.\left.-\left(A_{1}^{(1)}(t,-1)\right)^{T} \mathbf{B}_{1}(t,-1)\right)-\left(A_{1}^{(1)}(t, 1)\right)^{T} \mathbf{B}_{1}(t, 1)\right)
$$

$\left\{\frac{d}{d t}+I\right\}\left(\mathbf{B}_{2}(t, 1)-\mathbf{B}_{2}(t,-1)\right)=$

$$
\left.\left.-\left(A_{1}^{(1)}(t, 1)\right)^{T} \mathbf{B}_{1}(t, 1)\right)+\left(A_{1}^{(1)}(t,-1)\right)^{T} \mathbf{B}_{1}(t,-1)\right)
$$

Since the matrices $A_{1}^{(1)}(t, y)$ and $\mathbf{B}_{1}(t, y)$ are proportional to the parameter $y \in\{-1,1\}$, then the right-hand side of equation (65) is equal to zero, and therefore the trivial solution is the unique bounded solution of this equation. The equation (65) has a bounded solution if and only if its right-hand side is equal to zero after averaging. Hence, we find
$\Lambda_{2}=\lim _{T \rightarrow \infty} \frac{1}{T} \int_{0}^{T} \mathbf{B}_{1}^{T}(t, 1) A_{1}^{(1)}(t, 1) d t=\left(\begin{array}{cc}-\frac{35}{544} & \frac{63}{2720} \\ \frac{63}{2720} & -\frac{35}{544}\end{array}\right)$

Hence, for a sufficiently small $\varepsilon>0$, first moments of solutions of the equation (52) can be approximated by a vector function

$\mathbf{E}\{\mathbf{B}(t, y, \varepsilon) x(t, y, x)\}=\exp \left\{t A_{0}\right\} \exp \left\{t \Lambda_{2}\right\} \mathbf{E}\{x\}$

Matrix $\exp \left\{t A_{0}\right\}$ is periodic with respect to $t$, and eigenvalues of the matrix $\Lambda_{2}$ have negative real parts. Therefore, a vector function defined by (67) decreases exponentially at $t \rightarrow \infty$. Let us note that as the frequency of parametric perturbation of the oscillator (52) is equal to 2 and its self frequency is equal to 1 , there is a parametric resonance, and therefore any nontrivial solution of the equation (52) tends to infinity. Since the asymptotic behaviour of the first moments of solutions of the equation (52) and (67) is the same if $t \rightarrow \infty$, we can assert that for a sufficiently small $\varepsilon>0$, in spite of the parametric resonance, due to the Markov perturbations the amplitude of the first moments of solutions of the equation (52) tends exponentially to zero.

\section{REFERENCES}

Bogoljubov, N. N., Mitropoliskii, Ju. A., Samoilenko, A. M. (1976). Methods of Accelerated Convergence in Nonlinear Mechanics. Springer-Verlag, New York. 291 pp.

Dynkin, E. B. (1965). Markov Processes. Springer-Verlag, Berlin. 366 pp.

Fink, A. M. (1974). Almost periodic differential equations (Lecture Notes in Mathematics, No. 377). Berlin: Springer-Verlag. 336 pp.

Kato, T. (1980). Perturbation Theory for Linear Operators. SpringerVerlag, Berlin. 619 pp.

Skokos, Ch. (2010). The Lyapunov characteristic exponents and their computation. Lect. Notes Phys., 790, 63-135.

Received 27 September 2015

\section{LINEĀRU IMPULSVEIDA MARKOVA DINAMISKU SISTĒMU AR GANDRĪZ KONSTANTIEM KOEFICIENTIEM REDUCĒJAMĪBA, IZMANTOJOT SISTĒMAS MOMENTUS}

Šajā rakstā pētītas lineāras impulsveida dinamiskas sistēmas telpā $\mathbb{R}^{d}$, kuru parametri ir atkarīgi no ergodiska, gabaliem konstanta Markova procesa ar vērtībām telpā $Y, k a ̄$ arī no mazā parametra $\varepsilon$. Intervālos, kuros Markova process $x(t, y) \in \mathbb{R}^{d}$ saglabā konstantas vērtības, fāzu trajektorijas apmierina lineāru diferenciālvienādojumu sistēmu, kuras koeficienti ir tuvi konstantēm. Markova procesa pārslēguma laika momentos šìs trajektorijas mainās ar lēcienu, kura lielums ir lineāri atkarīgs no fāzu koordinātas un proporcionāls mazajam parametram $\varepsilon$. Rakstā piedāvāta metode un algoritms tādas bāzes $\mathbf{B}(t, y)$ izvēlei telpā $\mathbb{R}^{d}$, kas ļauj aproksimēt fāzu trajektoriju vidējās vērtības $\mathbf{E}\{x(t, y)\}$ ar lineāras diferenciālvienādojumu sistēmas ar konstantiem koeficientiem atrisinājumu. 